\title{
Chemical Decomposition of the TFSI Anion Under Aqueous Basic Conditions
}

Arthur France-Lanord, ${ }^{\dagger \dagger}$ Fabio Pietrucci, A. Marco Saitta,

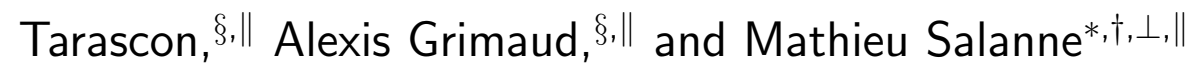

$\dagger$ †orbonne Université, CNRS, Physico-chimie des Electrolytes et Nanosystèmes Interfaciaux, PHENIX, F-75005 Paris, France

$\ddagger$ Sorbonne Université, Institut des Sciences du Calcul et des Données, ISCD, F-75005

Paris, France

ๆSorbonne Université, Muséum National d'Histoire Naturelle, UMR CNRS 7590, Institut de Minéralogie, de Physique des Matériaux et de Cosmochimie, IMPMC, F-75005 Paris, France

$\S$ Chimie du Solide et de l'Énergie, Collège de France, Paris, France

\|Réseau sur le Stockage Electrochimique de l'Energie (RS2E), FR CNRS 3459, France

$\perp$ Institut Universitaire de France (IUF), 75231 Paris Cedex 05, France

E-mail: mathieu.salanne@sorbonne-universite.fr 


\begin{abstract}
Understanding the interfacial reactivity of aqueous electrolytes is crucial for their use in future batteries. We investigate the reactivity of the bis(trifluoromethane)sulfonimide (TFSI) anion when exposed to a strong alkaline medium, by means of $a b$ initio molecular dynamics and enhanced sampling techniques. In particular, we study the nucleophilic attack by the hydroxide anion, which was proposed as a mechanism for the formation of the solid electrolyte interphase at the negative electrode with water-insalt electrolytes. While in the gas phase we recover a stable gaseous product, namely fluoroform, we observe the formation of trifluoromethanol in strong basic conditions, which then rapidly deprotonates to form $\mathrm{CF}_{3} \mathrm{O}^{-}$. This anion was suggested recently as a key compound leading to the formation of a solid electrolyte interphase on an Si-C anode. Such an approach could be leveraged to discover convenient additives leading to the formation of a stable interphase.
\end{abstract}

\title{
Introduction
}

In recent years, the research on aqueous batteries has been boosted by the development of water-in-salt electrolytes and their derivatives. ${ }^{1-3}$ They enable the application of large voltages, by pushing both the anodic and the cathodic limits much further than the ones of conventional aqueous solutions. On the negative electrode side, this is due to the formation of a stable solid electrolyte interphase (SEI) on some materials such as $\mathrm{Mo}_{6} \mathrm{~S}_{8},{ }^{1}$ but the accessible voltage window remains too far away for the conventional graphite anode. In addition, the SEI formed on the electrode was found to not be able to protect the battery against continuous electrolyte degradation during both cycling and storage. ${ }^{4}$ Much work therefore remains to be done before the development of a practical water-in-salt electrolyte based battery. ${ }^{5}$

The archetypal water-in-salt is lithium bis(trifluoromethane)sulfonimide (LiTFSI) at a molality of $21 \mathrm{~m}$. Under such conditions, the water molecules to Li ratio is largely below 
the cation coordination number under infinite dilution conditions (4), so that the water molecules compete to participate to their solvation shells. ${ }^{1}$ From the dynamics point of view, the properties of such electrolytes resemble the ones of ionic liquids. ${ }^{6}$ The formation of the SEI on the anode is due to the decomposition of the anion. Although first reports interpreted it as a direct reduction of TFSI, ${ }^{7}$ it was also shown that the water molecules were involved. Indeed, the formation of $\mathrm{H}_{2}$ showed that the hydrogen evolution reaction occurs, which leads to the formation of hydroxide anions in the vicinity of the electrode. ${ }^{8-10}{\mathrm{The} \mathrm{OH}^{-}}^{-}$ can chemically react with TFSI and catalyze the formation of a fluorinated SEI. ${ }^{8}$ However, a recent X-ray diffraction study, in which electrons are produced at the solid/electrolyte interface, concluded on a surface-reduction of TFSI in which no signs of $\mathrm{OH}^{-}$formation were observed. ${ }^{11}$ The mechanism of SEI formation in water-in-salt therefore remains to be fully understood.

Knowing the decomposition process of TFSI would certainly advance the feasibility of aqueous batteries. One important point is whether the anion directly reacts to form $\mathrm{CF}_{x^{-}}$ containing species and LiF, or whether reaction intermediates are involved. Indeed, it could allow to design additives in order to control the growth and the stability of the SEI, as well as to trigger it in the case of other material chemistries. Here we show using density functional theory (DFT)-based molecular dynamics (MD) that the trifluoromethanolate compound can be a key reaction intermediate. We study the reactivity of TFSI in a strongly basic aqueous solution, previously investigated with experimental means, ${ }^{8}$ using enhanced sampling methods borrowed from prebiotic chemistry. ${ }^{12}$ In this method reaction coordinates based on the coordination matrix of the atoms are used, ${ }^{13}$ which forces chemical reactions to occur within the limited timescale of the simulations while allowing enough flexibility to obtain unpredicted chemical products. Our results show that contrarily to the gas phase in which $\mathrm{OH}^{-}$and TFSI react to form a well-known stable (gaseous) product, trifluoromethane (fluoroform), in liquid phase trifluoromethanol forms and then deprotonates. This molecule is known to be highly reactive, thus providing an interesting lead to the origin of the SEI 
formation in water-in-salts.

\section{Results and Discussion}

\section{Reactants}

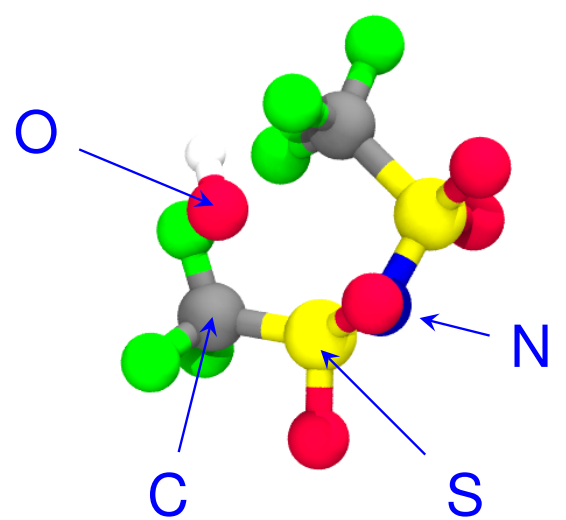

\begin{tabular}{|c|c|c|c|c|c|c|}
\hline & $\mathrm{S}$ & $\mathrm{N}$ & $\mathrm{F}$ & $\mathrm{O}$ & $\mathrm{C}$ & $\mathrm{H}$ \\
\hline $\mathrm{S}$ & 0 & 1 & 0 & 2 & 1 & 0 \\
\hline $\mathrm{N}$ & 2 & 0 & 0 & 0 & 0 & 0 \\
\hline $\mathrm{O}$ & 0 & 0 & 0 & 0 & 0 & 1 \\
\hline $\mathrm{C}$ & 1 & 0 & 3 & 0 & 0 & 0 \\
\hline
\end{tabular}

Reference pattern 1

\section{Products}
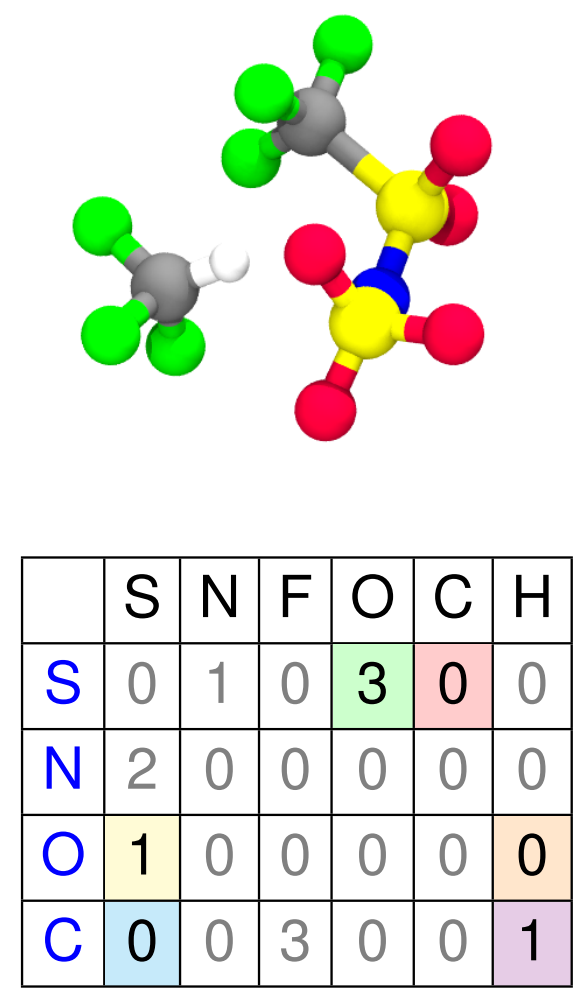

Reference pattern 2

Figure 1: Initial hypothesis of reactants and products, along with coordination tables used in path collective variables. Special atoms corresponding to row entries of the coordination tables are labeled in blue in the reactant state.

The main simulated system consists in a TFSI anion inside a basic aqueous LiOH electrolyte. Indeed simulating directly the water-in-salt would require too large simulation cells, and experiments have showed that the TFSI decomposes quickly under such conditions. ${ }^{8}$ DFT-based MD is the method of choice for studying chemical reactions in the liquid phase, 
but it requires biasing the system along reaction coordinates in order to overcome the free energy barriers. ${ }^{14}$ In the present work, two reaction coordinates are built by determining the coordination table of a selection of atoms in the reactants and products states. The former is easily chosen since the initial compounds are known. Concerning the products, based on the larger Mulliken charge carried by the sulfur atom within TFSI, ${ }^{15,16}$ a nucleophilic attack of $\mathrm{OH}^{-}$on this site was suggested. ${ }^{8}$ Weakening of the $\mathrm{C}-\mathrm{S}$ bond by $\mathrm{OH}^{-}$in an $\mathrm{Mg}(\mathrm{TFSI})_{2}-$ diglyme electrolyte was also investigated previously. ${ }^{17}$ We therefore chose trifluoromethane and the $\mathrm{N}\left(\mathrm{SO}_{2} \mathrm{CF}_{3}\right) \mathrm{SO}_{3}$ anion as the probable products of this first step, as illustrated on Figure 1 (which also gives the coordination tables for the two states). The first reaction coordinate $(s)$ follows the advancement of the reaction, while the second one $(z)$ allows the system to deviate from the direct pathway between the reactants and products states (see Supplementary Section 4 for a definition of $s$ and $z$ ). We then use metadynamics, ${ }^{18}$ an enhanced sampling method which allows to progressively escape from free energy minima. The main interest of this simulation setup is enforcing the system to react without having necessarily to reach the guessed products state: any other products can be formed. Once a reactive pathway is obtained, the free energy profile is computed using umbrella sampling.

In order to validate the method, the reaction was first enforced on isolated compounds, i.e. in the absence of any solvent or other species. The free energy profile obtained is shown on Figure 2A. It is clear that the method allows efficiently to pass easily from one state to the other. However, the free energy is large for the reactant state due to a significant contribution of the unscreened anion-anion repulsion. The products have the lowest free energy, confirming that they are a good guess for the final state. The reaction occurs through the approach of the $\mathrm{O}$ atom from the hydroxide to one of the $\mathrm{S}$ atoms from the TFSI, which leads to the breaking of the corresponding $\mathrm{C}-\mathrm{S}$ bond (see Supplementary movie 1). The carbon atom attracts the hydrogen from the hydroxide, resulting in the formation of a fluoroform molecule.

In the alkaline solution, the results are very different. The metadynamics does not bring 
A

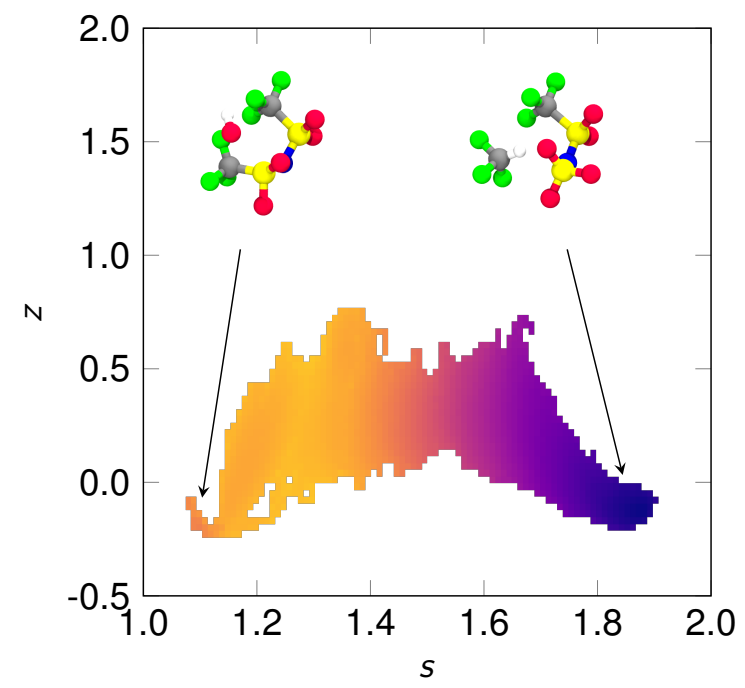

B

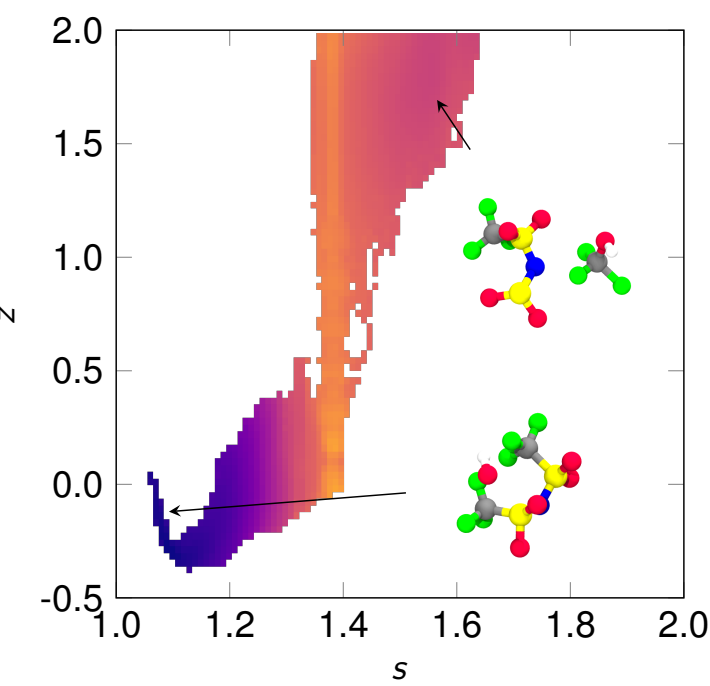

$F(\mathrm{~kJ} / \mathrm{mol})$

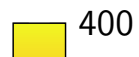

300

200

100

Figure 2: Free energy landscapes obtained from umbrella sampling, in the $(s, z)$ space. A: reaction in vacuum, $\mathbf{B}$ : reaction in the alkaline solution. An example of the geometry is shown for each metastable state, at $s \sim 1.1, s \sim 1.9$, and $s \sim 1.5$.

the system towards the same products, as shown on Figure 2B. Instead, it forms trifluoromethanol $\left(\mathrm{CF}_{3} \mathrm{OH}\right)$ and $\mathrm{N}\left(\mathrm{SO}_{2} \mathrm{CF}_{3}\right)\left(\mathrm{SO}_{2}\right)^{2-}$. Contrarily to the gas phase, the reactants display a lower free energy because the Coulombic interactions are screened by the presence of the electrolyte. The products are largely metastable, displaying a free energy of $206 \mathrm{~kJ} \mathrm{~mol}^{-1}$. Based on the converged free energy profile, the putative transition state (TS) has a free energy of $298 \mathrm{~kJ} \mathrm{~mol}^{-1}$. The largest statistical error on the free energy is of about $11 \mathrm{~kJ} \mathrm{~mol}^{-1}$, as explained in Supplementary Section 8. In order to determine precisely its position, we perform a committor analysis. This approach consists in picking several configurations along the transition pathway and performing a series of short (1 ps) simulations by randomly drawing initial velocities from the Maxwell-Boltzmann distribution. If the point is located before (resp. after) the TS, the trajectories will mostly evolve towards the reactant (resp. product) state, while close to the TS, they will share evenly between the two. The results for three points, as well as the structure of the deduced TS, are shown on Figure 3. Contrarily to the gas phase, the $\mathrm{OH}^{-}$reacts with the TFSI with a nucleophilic substitution mechanism. In the TS the three C-F bonds lie within the same plane, with the oxygen 
A
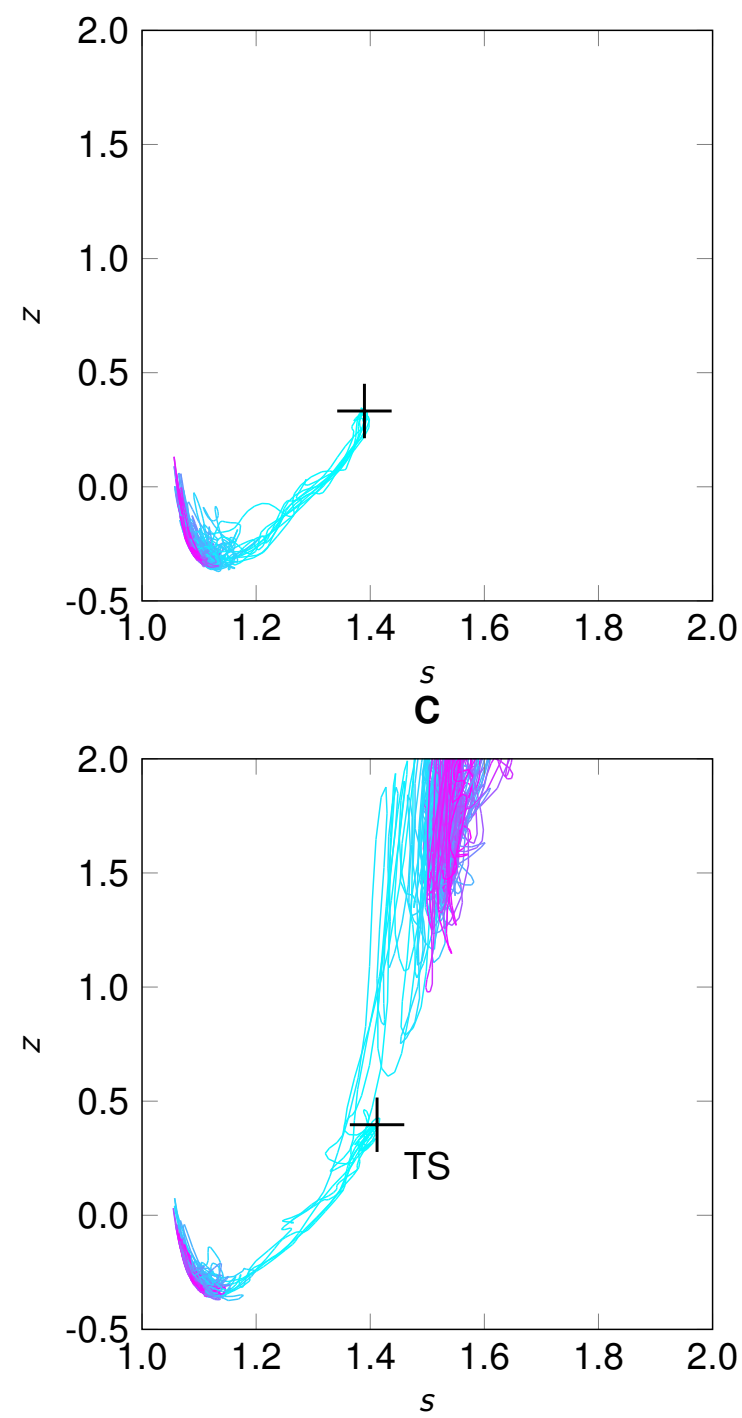

B

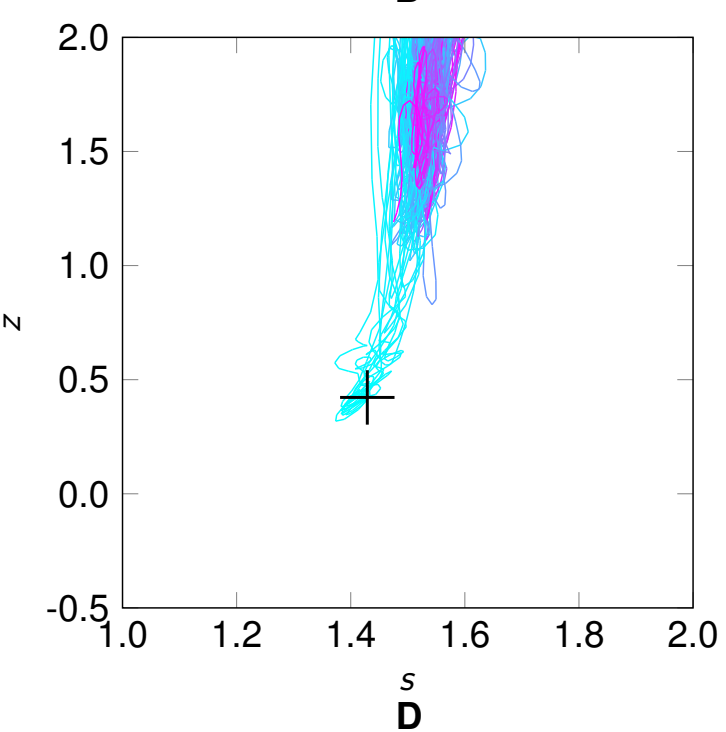

$t(\mathrm{ps})$

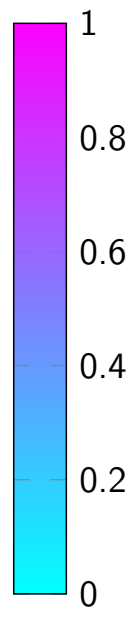

0.8

0.6

0.4

0.2

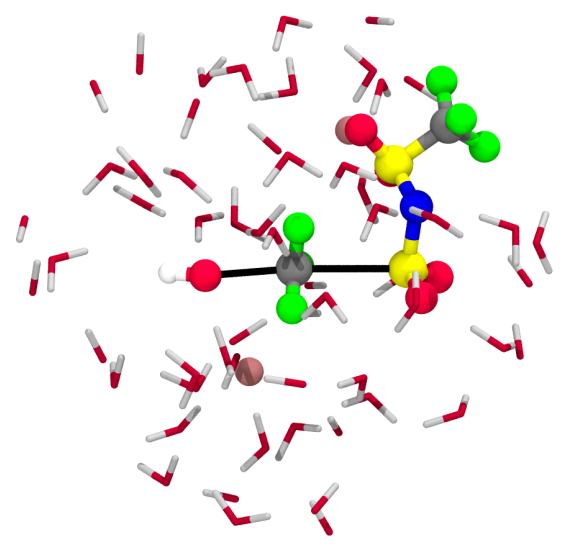

Figure 3: Ten independent trajectories starting from the same position in $(s, z)$ space (A: $s=1.39, z=0.33, \mathrm{~B}: s=1.43, z=0.42, \mathrm{C}: s=1.41, z=0.40)$, with randomized initial velocities. The color corresponds to the simulation time, going from cyan to magenta. The crosses denote the initial configuration positions in the $(s, z)$ space. The starting point in panel $\mathbf{C}$ corresponds to a member of the transition state ensemble. $\mathbf{D}$ : geometry of the identified transition state. Black lines emphasize the O-C and C-S distances, respectively $2.49 \AA$ and $3.21 \AA$.

and the sulfur atoms lying on the two sides of the plane (with corresponding C-O and C-S distances of $2.49 \AA$ and $3.21 \AA$ ). Supplementary movie 2 shows the reactive event.

It is then interesting to understand why this second reaction pathway is favored in the alkaline solution. A first hint would be that the $\mathrm{OH}^{-}$anions do not approach sufficiently 
A

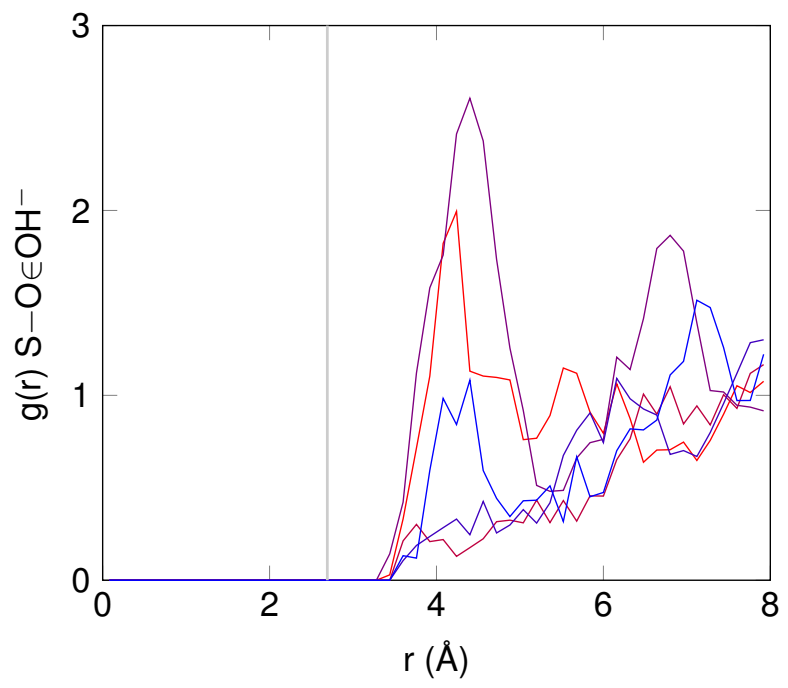

C

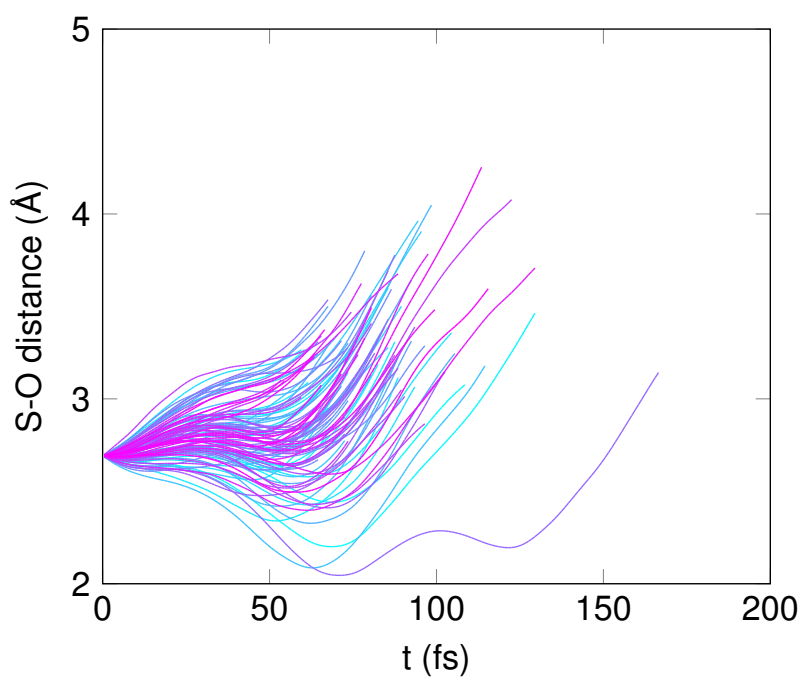

B

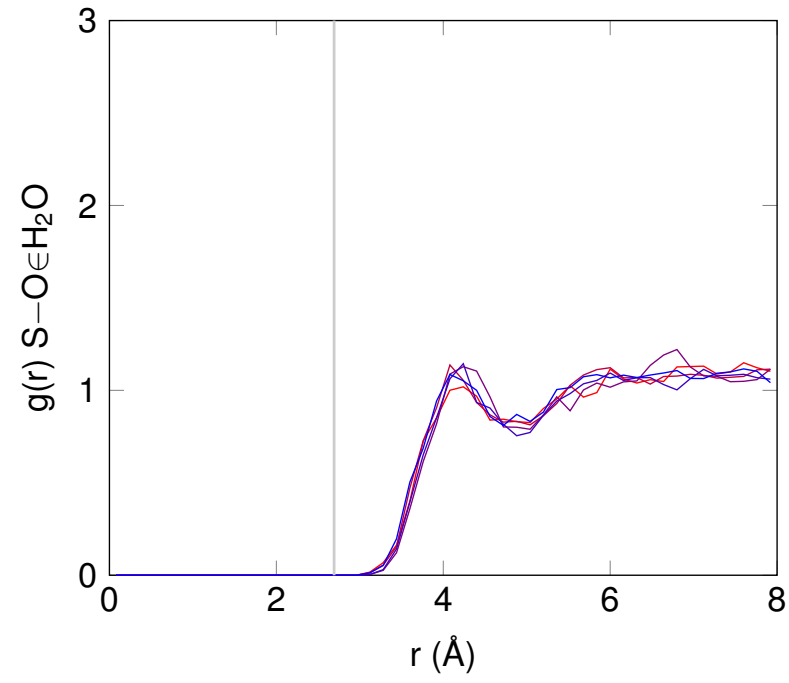

D

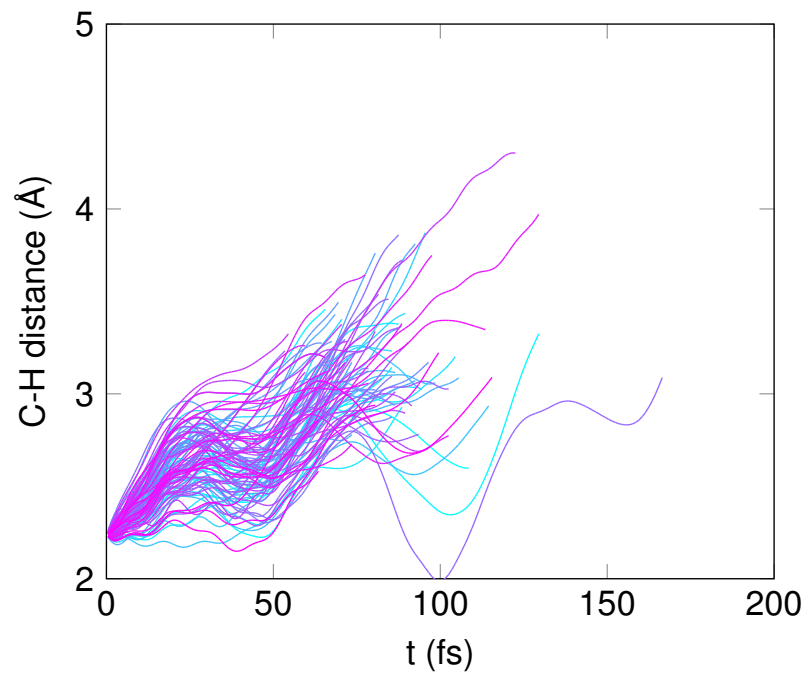

Figure 4: Top: radial distribution functions computed over unbiased equilibrium trajectories of the reactants in the alkaline solution, between the sulfur from TFSI and the oxygen from either hydroxide anions (A) or water molecules (B). Each color corresponds to an independent trajectory. Gray lines correspond to the distances in the gas phase transition state geometry. Bottom: distances between the sulfur from TFSI and the oxygen from $\mathrm{OH}^{-}$ $(\mathbf{C})$, and between the carbon from TFSI and the hydrogen from $\mathrm{OH}^{-}(\mathbf{D})$, as computed from the 100 committor trajectories starting from the solvated gas phase geometry. Each color corresponds to an independent trajectory.

the S-C bond. In order to check this point, we compute the radial distribution function $g(r)$ between the sulfur from TFSI, and the oxygen from either $\mathrm{OH}^{-}$or $\mathrm{H}_{2} \mathrm{O}$ molecules. These results are reported in Figures $4 \mathrm{~A}$ and B. As can be seen from the first peak located at 
$4 \AA$, both the hydroxide ions and water molecules are found in the surroundings of TFSI's $\mathrm{S}-\mathrm{C}$ bonds, although never as close as in the case of the gas phase TS (the corresponding distances are shown as gray lines in the two plots). This is also shown in Figure S5, which displays the shortest distances over the course of the trajectories. It is then necessary to understand why the hydroxide ions do not react with the TFSI. We thus attempted to force the formation of the expected trifluoromethane and $\mathrm{N}\left(\mathrm{SO}_{2} \mathrm{CF}_{3}\right) \mathrm{SO}_{3}$ products in the alkaline solution, by transferring the TS geometry obtained from the gas phase calculation to a liquid system. More details concerning the procedure are included in Supplementary Section 10. We simulated 100 committor trajectories from the gas phase TS in solution, with randomized velocities. In each case, the system relaxed to the reactants state, showing that this geometry is not anymore a good TS once immersed in the alkaline solution. This can be due to either energetic or entropic constraints on how reactant molecules must arrange to form the expected products. In Figure 4C (4D), we show the time-dependent distances between the sulfur (carbon) from TFSI and the oxygen (hydrogen) from $\mathrm{OH}^{-}$, over the course of the 100 committor trajectories mentioned above. Both distances overall increase over time. At short times $(t<25 \mathrm{fs})$, the hydrogen moves away from the TFSI faster than the oxygen, owing to its lighter mass, as well as to its propensity to form hydrogen bonds with water molecules. ${ }^{19}$ Consequently the hydroxide orientation becomes unfavorable for a nucleophilic attack to occur. On the contrary, to form the trifluoromethanol product, an $\mathrm{OH}^{-}$only needs to line up with the S-C bond, as can be seen in Supplementary movie 2 its hydrogen atom still keeps the freedom to form hydrogen bonds with the nearby water molecules.

We now discuss the reactivity of $\mathrm{CF}_{3} \mathrm{OH}$. Unlike the trifluoromethane which was initially envisaged, they do not form stable gaseous molecules. In the gas phase, trifluoromethanol eliminates HF in well-studied unimolecular reaction processes. ${ }^{20-23}$ It is however highly unlikely to observe such a reaction in an aqueous basic environment, in which trifluoromethanol should act as a proton donor. By performing further equilibrium simulations of $\mathrm{CF}_{3} \mathrm{OH}$, still 


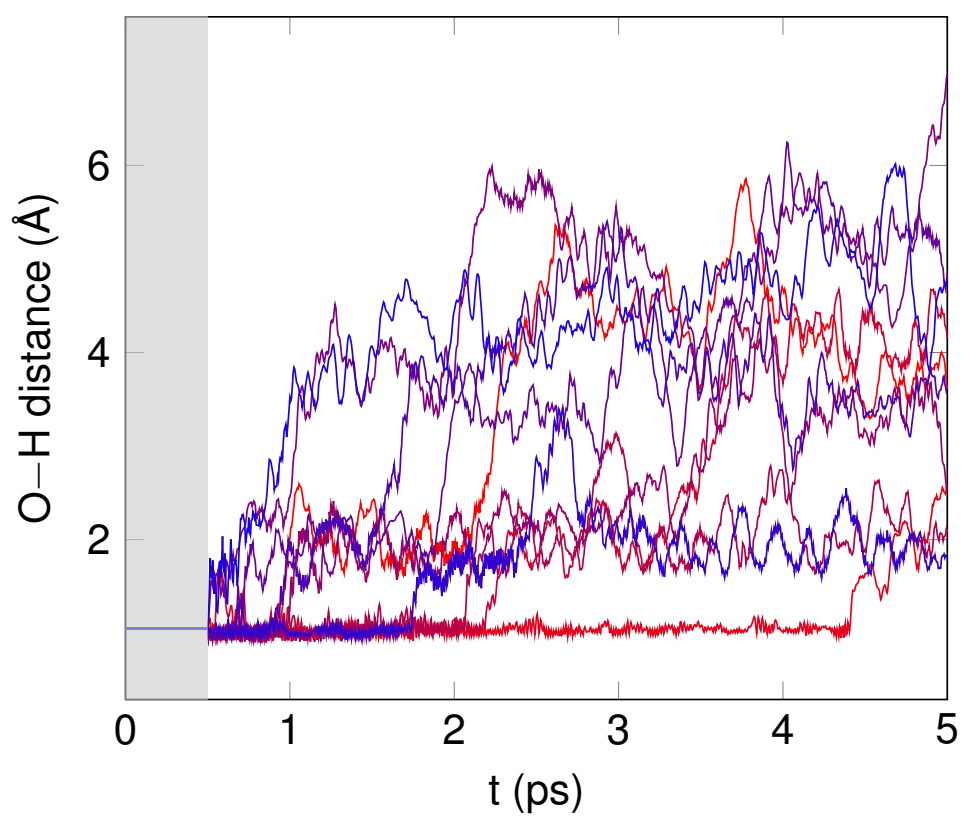

Figure 5: The $\mathrm{O}-\mathrm{H}$ distance in trifluoromethanol plotted as a function of simulation time, for ten independent trajectories. The gray box corresponds to the first $0.5 \mathrm{ps}$, in which the molecule's geometry is frozen. In all trajectories, the proton quickly leaves the molecule in order to form $\mathrm{CF}_{3} \mathrm{O}^{-}$.

in the alkaline solution, we observe a quick deprotonation to form the $\mathrm{CF}_{3} \mathrm{O}^{-}$anion, as shown on Figure 5. $\mathrm{CF}_{3} \mathrm{O}^{-}$reactivity towards a series of molecules was studied in the framework of atmospheric chemistry. ${ }^{24}$ In the gas phase, it was shown to react rapidly with many compounds $\left(\mathrm{ClONO}_{2}, \mathrm{HO}_{2} \mathrm{NO}_{2}, \mathrm{SO}_{2}\right.$, etc) by transferring fluoride, and it hydrolyzes in aqueous solution to form $\mathrm{F}^{-}, \mathrm{HF}$, and $\mathrm{CO}_{2}$. However, in the case of the water-in-salt and/or of the basic aqueous solution studied here, its reactivity may differ due to the presence of $\mathrm{Li}^{+}$ions, which is a strong Lewis acid (and is present under large concentrations). In a recent study on the use of dioxolone derivatives for high-energy-density lithium-ion batteries, the reaction

$$
\mathrm{CF}_{3} \mathrm{O}^{-}+\mathrm{Li}^{+} \longrightarrow \mathrm{CF}_{2} \mathrm{O}+\mathrm{LiF}
$$

was suggested as a key step for the formation of the SEI on a Si-C anode. ${ }^{25}$ It is therefore possible that this compound plays also a role in the formation of the SEI in water-in-saltbased aqueous ion batteries. 
In conclusion, we have shown that the TFSI anion reacts differently with the hydroxide anion in the gas phase and in aqueous basic solutions. In the gas phase, the reaction occurs through an attack of the C-S bond to form fluoroform, while in the liquid phase the hydrogen bond network hinders the hydroxide to orientate in order to form the new C-H bond. Instead, the reaction occurs via a nucleophilic attack on the carbon atom, leading to the formation of trifluoromethanol, which quickly loses its proton to form $\mathrm{CF}_{3} \mathrm{O}^{-}$. This species is a well-known fluorinating agent, so that its formation as a reaction intermediate provides an explanation of the composition of the SEI in water-in-salt based aqueous batteries. In future years, designing the SEI using well-targeted additives will be a key towards more efficient batteries with longer cycle life. Although trifluoromethanol is not a convenient solution in this respect because its synthesis requires complex conditions, ${ }^{26}$ finding other compounds that could generate it in situ during the first cycles of the battery would be an interesting lead for future research.

\section{Acknowledgements}

This work was supported by the French National Research Agency (Labex STORE-EX, Grant No. ANR-10-LABX-0076, and ANR BALWISE, Grant No. ANR-19-CE05-0014). This project has received funding from the European Research Council (ERC) under the European Union's Horizon 2020 research and innovation program (grant agreement no. 771294). This work was granted access to the HPC resources of CINES under the allocations A0100910463 and A0110811069 made by GENCI.

\section{References}

(1) Suo, L.; Borodin, O.; Gao, T.; Olguin, M.; Ho, J.; Fan, X.; Luo, C.; Wang, C.; Xu, K. "Water-in-Salt" Electrolyte Enables High-voltage Aqueous Lithium-ion Chemistries. Science 2015, 350, 938-943. 
(2) Yang, C.; Chen, J.; Qing, T.; Fan, X.; Sun, W.; von Cresce, A.; Ding, M. S.; Borodin, O.; Vatamanu, J.; Schroeder, M. A. et al. 4.0 V Aqueous Li-Ion Batteries. Joule 2017, 1, 122-132.

(3) Amiri, M.; Bélanger, D. Physicochemical and Electrochemical Properties of Water-inSalt Electrolytes. ChemSusChem 2021, 14, 2487-2500.

(4) Droguet, L.; Grimaud, A.; Fontaine, O.; Tarascon, J.-M. Water-in-Salt Electrolyte (WiSE) for Aqueous Batteries: A Long Way to Practicality. Adv. Ener. Mater. 2020, 10, 2002440.

(5) Jaumaux, P.; Yang, X.; Zhang, B.; Safaei, J.; Tang, X.; Zhou, D.; Wang, C.; Wang, G. Localized Water-in-Salt Electrolyte for Aqueous Lithium-Ion Batteries. Angew. Chem., Int. Ed. 2021, 60, 19965-19973.

(6) Li, Z.; Bouchal, R.; Mendez-Morales, T.; Rollet, A.-L.; Rizzi, C.; Le Vot, S.; Favier, F.; Rotenberg, B.; Borodin, O.; Fontaine, O. et al. Transport Properties of Li-TFSI Waterin-Salt Electrolytes. J. Phys. Chem. B 2019, 123, 10514-10521.

(7) Suo, L.; Oh, D.; Lin, Y.; Zhuo, Z.; Borodin, O.; Gao, T.; Wang, F.; Kushima, A.; Wang, Z.; Kim, H.-C. et al. How Solid-Electrolyte Interphase Forms in Aqueous Electrolytes. J. Am. Chem. Soc. 2017, 139, 18670-18680.

(8) Dubouis, N.; Lemaire, P.; Mirvaux, B.; Salager, E.; Deschamps, M.; Grimaud, A. The Role of the Hydrogen Evolution Reaction in the Solid-Electrolyte Interphase Formation Mechanism for "Water-in-Salt" Electrolytes. Energy Environ. Sci. 2018, 11, 3491-3499.

(9) Bouchal, R.; Li, Z.; Bongu, C.; Le Vot, S.; Berthelot, R.; Rotenberg, B.; Favier, F.; Freunberger, S.; Salanne, M.; Fontaine, O. Competitive Salt Precipitation/Dissolution During Free-Water Reduction in Water-in-Salt Electrolyte. Angew. Chem., Int. Ed. 2020, 59, 15913-15917. 
(10) Kim, Y.; Hong, M.; Oh, H.; Kim, Y.; Suyama, H.; Nakanishi, S.; Byon, H. R. Solid Electrolyte Interphase Revealing Interfacial Electrochemistry on Highly Oriented Pyrolytic Graphite in a Water-in-Salt Electrolyte. J. Phys. Chem. C 2020, 124, 20135-20142.

(11) Steinrück, H.-G.; Cao, C.; Lukatskaya, M.; Takacs, C.; Wan, G.; Mackanic, D.; Tsao, Y.; Zhao, J.; Helms, B.; Xu, K. et al. Interfacial Speciation Determines Interfacial Chemistry: X-ray-Induced Lithium Fluoride Formation from Water-in-salt Electrolytes on Solid Surfaces. Angew. Chem., Int. Ed. 2020, 59, 23180-23187.

(12) Saitta, A. M.; Saija, F. Miller Experiments in Atomistic Computer Simulations. Proc. Natl. Acad. Sci. U.S.A. 2014, 111, 13768-13773.

(13) Pietrucci, F.; Saitta, A. M. Formamide Reaction Network in Gas Phase and Solution via a Unified Theoretical Approach: Toward a Reconciliation of Different Prebiotic Scenarios. Proc. Natl. Acad. Sci. U.S.A. 2015, 112, 15030-15035.

(14) Branduardi, D.; Gervasio, F. L.; Parrinello, M. From $A$ to $B$ in Free Energy Space. $J$. Chem. Phys. 2007, 126, 054103.

(15) Benrabah, D.; Arnaud, R.; Sanchez, J.-Y. Comparative Ab Initio Calculations on Several Salts. Electrochim. Acta 1995, 40, 2437-2443.

(16) Rey, I.; Johansson, P.; Lindgren, J.; Lassègues, J. C.; Grondin, J.; Servant, L. Spectroscopic and Theoretical Study of $\left(\mathrm{CF}_{3} \mathrm{SO}_{2}\right)_{2} \mathrm{~N}^{-}\left(\mathrm{TFSI}^{-}\right)$and $\left(\mathrm{CF}_{3} \mathrm{SO}_{2}\right)_{2} \mathrm{NH}(\mathrm{HTFSI}) . J$. Phys. Chem. A 1998, 102, 3249-3258.

(17) Yu, Y.; Baskin, A.; Valero-Vidal, C.; Hahn, N. T.; Liu, Q.; Zavadil, K. R.; Eichhorn, B. W.; Prendergast, D.; Crumlin, E. J. Instability at the Electrode/Electrolyte Interface Induced by Hard Cation Chelation and Nucleophilic Attack. Chem. Mater. 2017, 29, 8504-8512. 
(18) Laio, A.; Parrinello, M. Escaping Free-Energy Minima. Proc. Natl. Acad. Sci. U.S.A. 2002, 99, 12562-12566.

(19) Marx, D.; Chandra, A.; Tuckerman, M. E. Aqueous Basic Solutions: Hydroxide Solvation, Structural Diffusion, and Comparison to the Hydrated Proton. Chem. Rev. 2010, 110, 2174-2216.

(20) Francisco, J. An Examination of Primary and Secondary Dissociation Pathways of Trifluoromethanol: Identification of Plausible Routes Leading to the Formation of Hydrogen Fluoride. Chem. Phys. 1991, 150, 19-27.

(21) Brudnik, K.; Wójcik-Pastuszka, D.; Jodkowski, J. T.; Leszczynski, J. Theoretical Study of the Kinetics and Mechanism of the Decomposition of Trifluoromethanol, Trichloromethanol, and Tribromomethanol in the Gas Phase. J. Mol. Model. 2008, 14, 1159-1172.

(22) Nguyen, M. T.; Matus, M. H.; Ngan, V. T.; Haiges, R.; Christe, K. O.; Dixon, D. A. Energetics and Mechanism of the Decomposition of Trifluoromethanol. J. Phys. Chem. A 2008, 112, 1298-1312.

(23) Gulvi, N. R.; Maliekal, P. J.; Mapari, M. G.; Badani, P. M. Theoretical Kinetic Analysis and Molecular Dynamic Simulations for Exploring the Dissociation Pathways of $\mathrm{CF}_{3} \mathrm{OH}$. Comput. Theor. Chem. 2021, 1194, 113056.

(24) Huey, L. G.; Villalta, P. W.; Dunlea, E. J.; Hanson, D. R.; Howard, C. J. Reactions of $\mathrm{CF}_{3} \mathrm{O}^{-}$with Atmospheric Trace Gases. J. Phys. Chem. 1996, 100, 190-194.

(25) Park, S.; Jeong, S. Y.; Lee, T. K.; Park, M. W.; Lim, H. Y.; Sung, J.; Cho, J.; Kwak, S. K.; Hong, S. Y.; Choi, N.-S. Replacing Conventional Battery Electrolyte Additives with Dioxolone Derivatives for High-Energy-Density Lithium-Ion Batteries. Nat. Commun. 2021, 12, 838. 
(26) Christe, K. O.; Hegge, J.; Hoge, B.; Haiges, R. Convenient Access to Trifluoromethanol. Angew. Chem., Int. Ed. 2007, 46, 6155-6158. 